\title{
Longitudinal collective modes in simple liquid binary alloys: A computer simulation study
}

\author{
N. Anento and J. A. Padró \\ Departament de Física Fonamental, Universitat de Barcelona, Diagonal, 647, 08028 Barcelona, Spain
}

(Received 18 May 2000)

\begin{abstract}
The dynamic collective properties of the liquid $\mathrm{Li}_{0.7}-\mathrm{Mg}_{0.3}$ alloy are studied by molecular-dynamics simulation. The dynamic structure factors and longitudinal current correlations at wave vectors between the hydrodynamic and kinetic regime $\left(0.2<k<4 \AA^{-1}\right)$ are analyzed. In order to discuss the influence of the mass difference between particles on the longitudinal modes, the same alloy, except that the mass of the heavy atoms was increased by a factor of 10 , was simulated. The resulting properties are compared with those of the ordinary $\mathrm{Li}_{0.7}-\mathrm{Mg}_{0.3}$ alloy. It is shown that at wave vectors prior to the hydrodynamic region both fast and slow longitudinal modes of kinetic character propagate through light and heavy particles, respectively. In the hydrodynamic limit fast and slow modes merge into a single acoustic mode. It is corroborated that fast propagating modes in disparate mass liquid mixtures have a kinetic character. The number-number and concentration-concentration time correlation functions were also determined. The former shows a behavior analogous to that of the corresponding function in one-component liquids. The second reflects the existence of propagating concentration modes.
\end{abstract}

\section{INTRODUCTION}

Molecular-dynamics (MD) simulation is a powerful tool to investigate the dynamic collective properties of liquids. MD is particularly useful to analyze these properties at intermediate wave vectors and frequencies, i.e., the same $k-\omega$ region that may be explored in neutron-scattering experiments. Recently, MD simulation has been extensively applied to the study of both the structure and the dynamic properties of liquid metals whereas liquid alloys have received less attention, especially in the case of dynamic collective properties. Since the pioneer MD simulation of Na-K liquid alloy ${ }^{1}$ the majority of studies have been centered on the alloy $\mathrm{Li}_{4} \mathrm{~Pb}$. In a MD simulation of this system Bosse et $a .^{2}{ }^{2}$ observed the existence of high-frequency peaks in the partial Li-Li dynamic structure factors at rather large wave vectors. These peaks were associated with longitudinal modes that propagate through light particles with a velocity much higher than that of the ordinary sound (the so-called "fast sound"). In recent years this topic has been the subject of controversy. High-frequency modes were detected by neutron-scattering experiments in $\mathrm{Li}_{4} \mathrm{~Pb}$ and $\mathrm{Li}_{4} \mathrm{Tl}$ alloys ${ }^{3,4}$ as well as in binary rare-gas fluid mixtures by both neutron scattering ${ }^{5}$ and MD simulation. ${ }^{6,7}$ In addition, the existence of high-frequency modes beyond the hydrodynamic regime in disparate mass binary mixtures was predicted by kinetic theory. ${ }^{8}$ Recent MD calculations have suggested that fast modes propagating through the light particles have a nonhydrodynamic (kinetic) character and they are accompanied by slow modes involving the heavy particles. ${ }^{9,10}$

One of the objectives of the present study is to analyze the dynamic collective properties of liquid $\mathrm{Li}-\mathrm{Mg}$ at wave vectors and frequencies between the hydrodynamic and kinetic regions. This system may be considered as representative of simple "ideal" alloys since it does show neither the ionic character of Li- $\mathrm{Pb}$ (Ref. 11) nor the tendency to phase separation of $\mathrm{Li}-\mathrm{Na} .{ }^{12}$ In particular, we have simulated the $\mathrm{Li}_{0.7} \mathrm{Mg}_{0.3}$ alloy. To our knowledge there is no experimental information or MD results on the dynamic collective properties of this alloy. The study will be based on the MD results obtained with an interatomic potential that reproduces the main structural features of Li-Mg alloys. ${ }^{13}$ Another objective is to investigate the relationship between the differences in the atomic masses of the species in the mixture and the existence of fast and slow propagating longitudinal modes as well as the character of these modes. For this purpose we have also simulated the $\mathrm{Li}_{0.7} \mathrm{Mg}_{0.3}^{*}$ alloy, which is identical to $\mathrm{Li}_{0.7} \mathrm{Mg}_{0.3}$ except that the mass of the $\mathrm{Mg}$ atoms was increased by a factor of 10 (they will be termed $\mathrm{Mg}^{*}$ ). Then, the mass ratio in this system $\left(m_{\mathrm{Mg}} * / m_{\mathrm{Li}}=35\right)$ is similar to that in the Li-Pb alloy $\left(m_{\mathrm{Pb}} / m_{\mathrm{Li}} \approx 30\right)$.

\section{COMPUTER SIMULATION DETAILS}

MD simulations of the $\mathrm{Li}_{0.7} \mathrm{Mg}_{0.3}$ alloy at $T=887 \mathrm{~K}$ and $\rho=4.071 \times 10^{-2} \AA^{-3}$ were carried out. The system was made up of 570 particles enclosed in a cubic box with ordinary periodic boundary conditions. Effective interatomic pair potentials obtained according to the neutral pseudoatom method $^{14}$ were used. These potentials, which are free of adjustable parameters, describe accurately the structure of liquid $\mathrm{Li},{ }^{15,16} \mathrm{Li}-\mathrm{Na}$, and $\mathrm{Li}-\mathrm{Mg},{ }^{13}$ and were successfully applied to the MD study of the dynamic collective properties of liquid $\mathrm{Li}$ (Ref. 16) and Li-Na. ${ }^{17}$

The partial intermediate scattering functions $\left[F^{i j}(k, t) ; j\right.$ $=1,2 ; j=1,2 ; 1 \equiv \mathrm{Li}, 2 \equiv \mathrm{Mg}]$ as well as the partial longitudinal current correlation functions $\left[C_{L}^{i j}(k, t)\right]$ were calculated during the MD simulation runs according to the usual definitions. ${ }^{18,19}$ These properties were determined for 26 wave vectors ranging from $k=0.26 \AA^{-1}$ to $k=3.97 \AA^{-1}$, compatible with the periodic boundary conditions. The frequency-dependent spectra $\left[S^{i j}(k, \omega), C_{L}^{i j}(k, \omega)\right]$ were obtained by Fourier transforming the corresponding timedependent correlation functions. We have also analyzed the number density-concentration correlation functions, defined $\mathrm{as}^{20}$ 

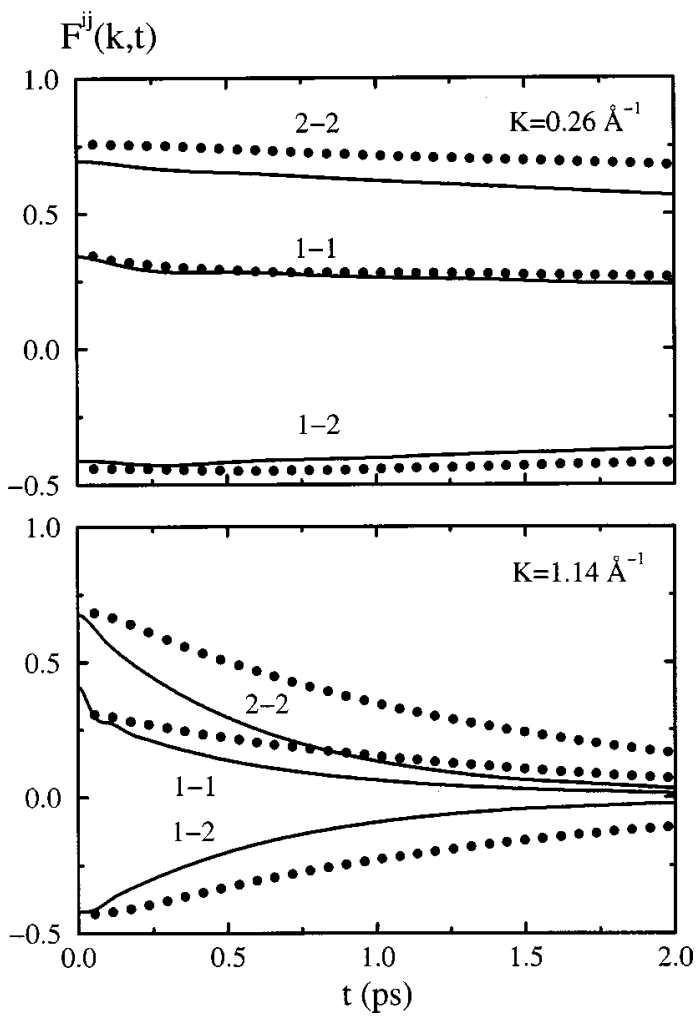

FIG. 1. Partial intermediate scattering functions for the $\mathrm{Li}_{0.7} \mathrm{Mg}_{0.3}$ (lines) and $\mathrm{Li}_{0.7} \mathrm{Mg}_{0.3}^{*}$ (dots) alloys.

$$
\begin{gathered}
F^{N N}(k, t) \equiv x_{1} F^{11}(k, t)+x_{2} F^{22}(k, t)+2\left(x_{1} x_{2}\right)^{1 / 2} F^{12}(k, t), \\
F^{N C}(k, t) \equiv x_{1} x_{2}\left[F^{11}(k, t)-F^{22}(k, t)+\left(x_{2}-x_{1}\right)\right. \\
\left.\times\left(x_{1} x_{2}\right)^{-1 / 2} F^{12}(k, t)\right], \\
F^{C C}(k, t) \equiv x_{1} x_{2}\left[x_{2} F^{11}(k, t)+x_{1} F^{22}(k, t)\right. \\
\left.-2\left(x_{1} x_{2}\right)^{1 / 2} F^{12}(k, t)\right],
\end{gathered}
$$

where $x_{1}$ and $x_{2}$ are the molar fractions of species 1 and 2, respectively. Analogous definitions were used for the other correlation functions and corresponding spectra. It should be noted that the $N N$ correlations represent the averaged behavior of the system and in the limiting case of a mixture of two identical species they are identical to the correlation functions directly calculated for the pure liquid.

We also performed MD simulations of the same system at the same temperature and atomic density but assuming that the atomic mass of the heavier atoms $\left(2 \equiv \mathrm{Mg}^{*}\right)$ is ten times larger than that of magnesium $\left(m_{\mathrm{Mg} *}=10 m_{\mathrm{Mg}}\right)$. The interaction potentials, MD procedure, and calculated properties were the same as for $\mathrm{Li}_{0.7}-\mathrm{Mg}_{0.3}$. However, the $k$-dependent properties for $\mathrm{Li}_{0.7} \mathrm{Mg}_{0.3}^{*}$ were only obtained up to $k$ $=1.48 \AA^{-1}$ and special attention was paid to the results at low wave vectors. So, an additional MD simulation using 6000 particles allowed us to determine the collective properties down to $k=0.12 \AA^{-1}$. We checked that properties for very similar wave vectors in the low- $k$ region obtained from MD simulations with either 570 or 6000 particles were in good agreement.

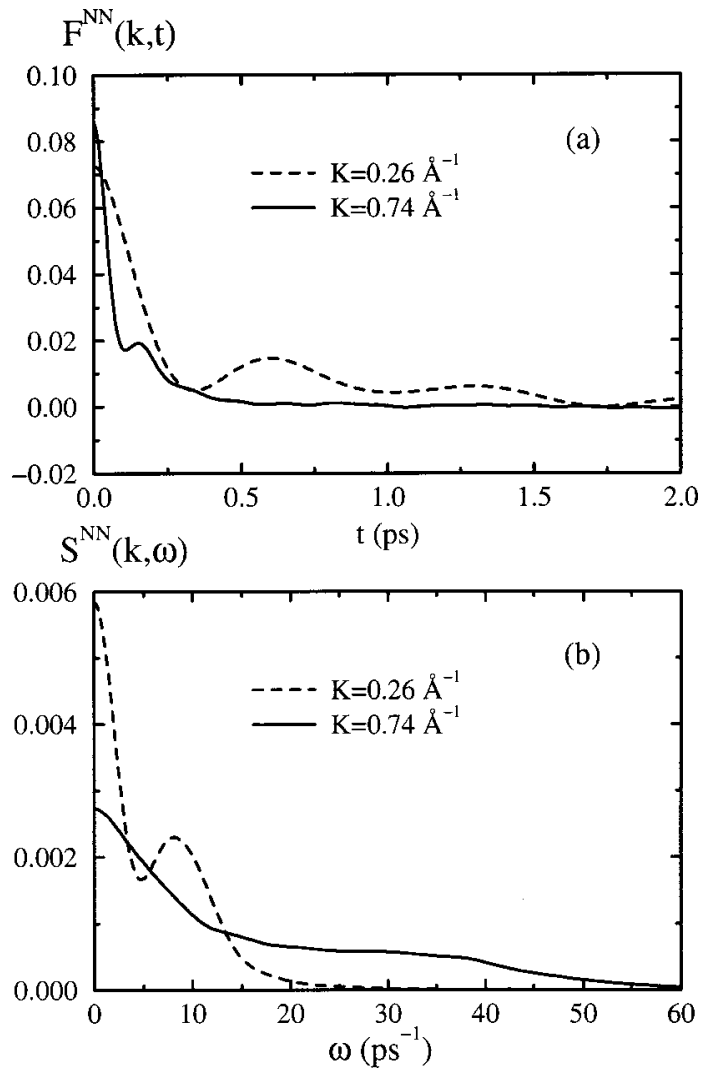

FIG. 2. Number-number intermediate scattering functions (a) and dynamic structure factors (b) for the $\mathrm{Li}_{0.7} \mathrm{Mg}_{0.3}$ alloy.

\section{DYNAMIC STRUCTURE FACTORS}

\section{A. $\mathbf{L i}_{0.7} \mathbf{M g}_{0.3}$ system}

Figure 1 shows the partial intermediate scattering functions $\left[F^{i j}(k, t)\right]$ obtained for two representative wave vectors in the low- $k$ region. $F^{12}(k, t)$ is negative and the three functions go monotonically to zero, showing slower decays as $k$ decreases. The long-time behavior of the $F^{i j}(k, t)$ functions should be reflected in the Rayleigh peaks at $\omega=0$ of the corresponding dynamic structure factors $\left[S^{i j}(k, \omega)\right]$. In the hydrodynamic limit, the Rayleigh peak of the dynamic structure factor $[S(k, \omega)]$ for pure liquids represents a nonpropagating thermal mode. ${ }^{18}$ In these systems the collective mass currents are zero ${ }^{21}$ and the decay of the $F(k, t)$ 's at low $k$ 's are quite fast [see, for example, the $F(k, t)$ 's for liquid $\mathrm{Li}$ (Ref. 16)]. However, the Rayleigh peak in binary liquids is related to both heat conduction and diffusion as well as to coupling effects. ${ }^{21,22}$ This should be the origin of the large discrepancies between $F^{i j}(k, t)$ and $F(k, t)$ at low $k$ 's. The slow decays of the former should then be mainly associated with diffusion effects.

The $F^{N C}(k, t)$ and $F^{C C}(k, t)$ correlation functions at low $k$ 's show persistent long-time decays analogous to those of the $F^{i j}(k, t)$ 's. Nevertheless, the $F^{N N}(k, t)$ 's are one order of magnitude lower than the $F^{i j}(k, t)$ 's and decrease much faster with time [see Figs. 1 and 2(a)]. This should be attributed to large cancellations between the three partial functions since $F^{12}(k, t)$ is of similar magnitude but opposite sign than $F^{11}(k, t)$ and $F^{22}(k, t)$. The behavior of $F^{N N}(k, t)$ is similar to that of $F(k, t)$ for pure liquids. $F^{N N}(k, t)$ at $k$ 
$=0.26 \AA^{-1}$ shows marked oscillations which become more damped at smaller $k$ 's being practically reduced to a single peak at $k=0.74 \AA^{-1}$ [Fig. 2(a)]. This is in agreement with the oscillating $F^{N N}(k, t)$ functions found for the K-Cs alloy at low $k$ 's. ${ }^{23}$ Unlike the partial $F^{i j}(k, t)$ 's, $F^{N N}(k, t)$ goes to zero at rather short times and $S^{N N}(k, \omega)$ could be reliably calculated. The $S^{N N}(k, \omega)$ 's at the lowest $k$ 's explored in this study show a clear Rayleigh-Brillouin structure similar to that of $S(k, \omega)$ for pure liquids near the hydrodynamic regime. ${ }^{18,19}$ As $k$ increases, the $S^{N N}(k, \omega)$ Brillouin peak becomes more poorly resolved and for wave vectors between $k \approx 0.4 \AA^{-1}$ and $k \approx 1.5 \AA^{-1}$ only remnant shoulders are noticed [Fig. 2(b)]. No significant peaks or shoulders can be observed for $k$ higher than $\approx 1.5 \AA^{-1}$ (Fig. 3).

$S^{N N}(k, \omega)$ at $k=0.26 \AA^{-1}$ show a marked Brillouin peak at $\omega_{B} \approx 8.1 \mathrm{ps}^{-1}$ [Fig. 2(b)] that may be associated with propagating acoustic modes through the mixture. The velocity of propagation of these modes can be calculated according to the relation $c_{s}=\omega_{B} / k$. Thus, the estimated value of the adiabatic sound velocity in $\mathrm{Li}_{0.7} \mathrm{Mg}_{0.3}$ is $c_{s}^{\mathrm{LiMg}} \approx 3.1$ $\times 10^{3} \mathrm{~m} \mathrm{~s}^{-1}$. To our knowledge this velocity has not been experimentally determined. We can compare our result with that from an earlier MD simulation of liquid $\mathrm{Li}$ at similar conditions $\left(T=843 \mathrm{~K}\right.$ and $\left.\rho=4.162 \times 10^{-2} \AA^{-3}\right)$ and also using an effective potential obtained by the neutral pseudoatom method. The $c_{s}$ value obtained in one such MD study, ${ }^{16}$ $c_{s}^{\mathrm{Li}}=4.3 \times 10^{3} \mathrm{~m} \mathrm{~s}^{-1}$, is in good agreement with the experimental value. If one assumes that in the hydrodynamic limit the mixture is equivalent to a pure liquid made up of particles with an average atomic mass $m_{\mathrm{Li}-\mathrm{Mg}}=0.7 m_{\mathrm{Li}}$ $+0.3 m_{\mathrm{Mg}}$ and uses a simple mass scaling law, ${ }^{23}$ one obtains: $c_{s}^{\mathrm{LiMg}} \approx c_{s}^{\mathrm{Li}} \quad\left(m_{\mathrm{Li}} / m_{\mathrm{Li}-\mathrm{Mg}}\right)^{1 / 2}=3.2 \times 10^{3} \mathrm{~m} \mathrm{~s}^{-1} . \quad$ This value is very close to that estimated from $\omega_{B}$ in $S^{N N}(k, \omega)$ at $k$ $=0.26 \AA^{-1}$.

The calculation of the three partial dynamic structure factors $S^{i j}(k, \omega)$ can provide interesting information on the microscopic mechanisms of propagating longitudinal modes. So, for example, it was suggested that the peaks displayed by $S^{11}(k, \omega)$ in $\mathrm{Li}_{4} \mathrm{~Pb}$ can be associated with propagating fast modes supported by the light particles alone. ${ }^{2}$ However, the low- $k S^{i j}(k, \omega)$ factors for $\mathrm{Li}_{0.7} \mathrm{Mg}_{0.3}$ can hardly be obtained due to the slow decays of the $F^{i j}(k, t)$ 's. Since the influence on $F^{N N}(k, t)$ [and therefore on $S^{N N}(k, \omega)$ ] of the long-time part of the $F^{i j}(k, t)$ 's is very weak we tried to remove it. According to viscoelastic theory, which was successfully applied to Li-Na alloy, ${ }^{17}$ the long-time decay of $F^{i j}(k, t)$ may be approximately represented by an exponential function. Thus, we defined

$$
F_{-}^{i j}(k, t)=F^{i j}(k, t)-A_{i j} \exp \left(-\alpha_{i j} t\right),
$$

where $A_{i j}$ and $\alpha_{i j}$ are $k$-dependent constants that were determined by fitting $A_{i j} \exp \left(-\alpha_{i j} t\right)$ to the long-time part of $F^{i j}(k, t)$ for a given $k$. We checked that the values of the resulting constants were quite close to those directly calculated according to the viscoelastic approximation. As expected, the new $F_{-}^{i j}(k, t)$ function keeps both the oscillatory and the fast-decaying components of $F^{i j}(k, t)$ whereas the slow-decaying component is practically absent. These $F_{-}^{i j}(k, t)$ functions were Fourier transformed to obtain the
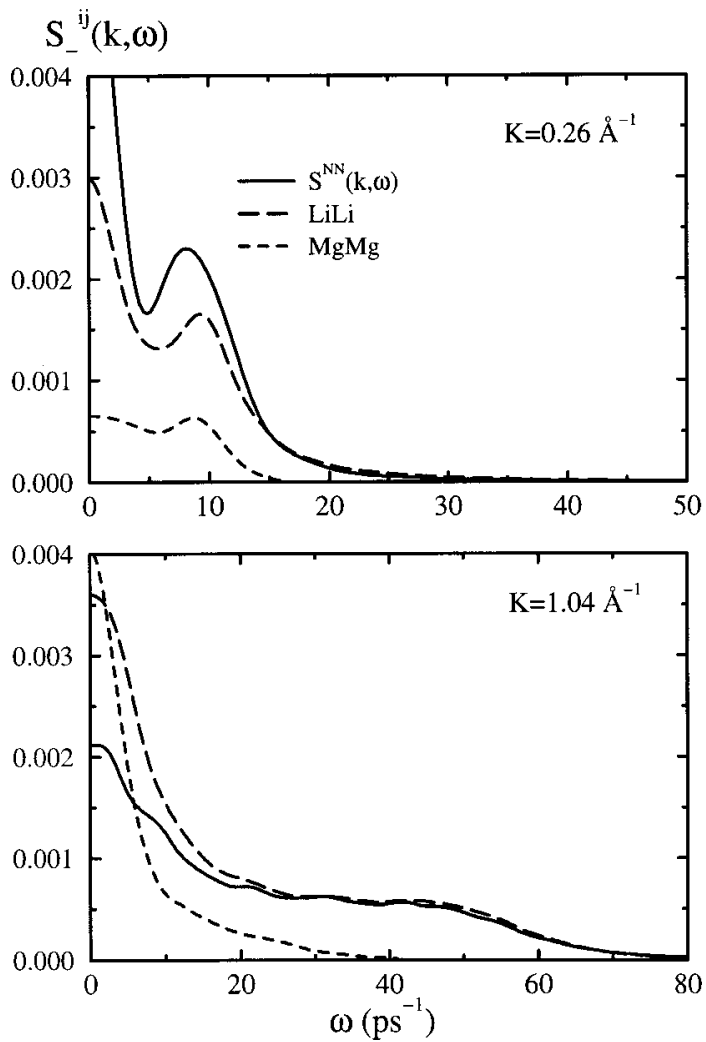

FIG. 3. Corrected partial dynamic structure factors $S_{-}^{11}(k, \omega)$ and $S_{-}^{22}(k, \omega)$ (as defined in the text) and number-number dynamic structure factors $S^{N N}(k, \omega)$ for the $\mathrm{Li}_{0.7} \mathrm{Mg}_{0.3}$ alloy.

corresponding $S_{-}^{i j}(k, \omega)$ 's. It is interesting to observe that both $S_{-}^{11}(k, \omega)$ and $S_{-}^{22}(k, \omega)$ as well as $S^{N N}(k, \omega)$ for $k$ $=0.26 \AA^{-1}$ show pronounced peaks at very similar frequencies (Fig. 3). This behavior should be typical of the partial dynamic structure factors in the hydrodynamic region, i.e., for a given $k$, the characteristic frequency of the two species in the mixture is the same and corresponds to the propagating sound mode. Nevertheless, there are no indications of any noticeable peak or shoulder in the $S_{-}^{22}(k, \omega)$ functions beyond $k \approx 0.4 \AA^{-1}$. This shows that the high-frequency shoulders of the $S^{N N}(k, \omega)$ 's in this $k$ region should be associated only with the light particles (see the results for $k$ $=1.04 \AA^{-1}$ in Fig. 3), which is consistent with the earlier results for liquid $\mathrm{Li}_{4} \mathrm{~Pb}^{2,9}$

Beginning from $k \approx 2 \AA^{-1}$, the cross $F^{12}(k, t)$ function increases with $k$ and becomes positive at $k \approx 2.2 \AA^{-1}$. At $k$ $=2.44 \AA^{-1}$ the three $F^{i j}(k, t)$ functions show their maximum initial values and $S^{N N}(k, \omega)$ has a narrow peak at $\omega$ $=0$ that becomes lower and broader for neighboring values of $k$ (either smaller or larger) (Fig. 4). This behavior, which is analogous to that of $S(k, \omega)$ for pure liquids at wavelengths corresponding to the maximum of the static structure factor (de Gennes narrowing), ${ }^{18,19}$ should be attributed to the strong spatial correlations at $k=2.44 \AA^{-1}$. It should be pointed out that the three partial static structure factors $S^{i j}(k)$, and then $S^{N N}(k)$, for $\mathrm{Li}_{0.7} \mathrm{Mg}_{0.3}$ have their first peaks located at very similar wave vectors $\left(k \approx 2.4 \AA^{-1}\right)$. This is consistent with the rather small differences in the "structural characteristics" (atomic size, interatomic potentials) of the two species in this mixture. ${ }^{13}$ 


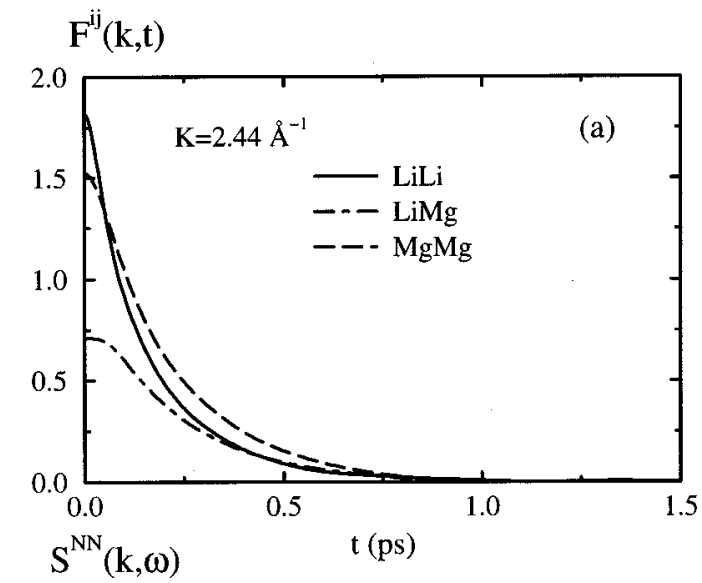

\section{B. $\mathrm{Li}_{0.7} \mathrm{Mg}_{0.3}^{*}$ system}

The main trends of the partial $F^{i j}(k, t)$ functions for $\mathrm{Li}_{0.7} \mathrm{Mg}_{0.3}^{*}$ are similar to those for $\mathrm{Li}_{0.7} \mathrm{Mg}_{0.3}$ (Fig. 1). The most significant discrepancy is that $F^{i j}(k, t)$ for the former system shows more persistent time decays than for the second, especially in the case of $F^{22}(k, t)$, which is consistent with the bigger mass of $\mathrm{Mg}^{*}$. Nevertheless, the $F^{11}(k, t)$ functions for $\mathrm{Li}_{0.7} \mathrm{Mg}_{0.3}^{*}$ are markedly different from those for $\mathrm{Li}_{4} \mathrm{~Pb}$. For $\mathrm{Li}_{4} \mathrm{~Pb}$ they show small initial values and quite fast oscillatory decays while their $S^{11}(k, \omega)$ spectra present noticeable Brillouin peaks that reveals the existence of highfrequency modes. ${ }^{2,9}$ On the contrary, the $F^{11}(k, t)$ functions for $\mathrm{Li}_{0.7} \mathrm{Mg}_{0.3}^{*}$ show initial values one order of magnitude higher and much more slower decays than for $\mathrm{Li}_{4} \mathrm{~Pb}$. Then, possible oscillations in $F^{11}(k, t)$ and corresponding $S^{11}(k, \omega)$ peaks remain unseen. These findings prove that the large discrepancies between the $F^{i j}(k, t)$ 's for both $\mathrm{Li}_{0.3} \mathrm{Mg}_{0.3}$ and $\mathrm{Li}_{0.7} \mathrm{Mg}_{0.3}^{*}$ with regard to those for $\mathrm{Li}_{4} \mathrm{~Pb}$ cannot be attributed only to the differences between the atomic masses of species in the mixtures.

As with $\mathrm{Li}_{0.7} \mathrm{Mg}_{0.3}$, the slow long-time decays of the three $F^{i j}(k, t)$ partials for $\mathrm{Li}_{0.7} \mathrm{Mg}_{0.3}^{*}$ are largely cancelled when $F^{N N}(k, t)$ is calculated. At small $k$ 's, $F^{N N}(k, t)$ show oscillations (smoother than for $\mathrm{Li}_{0.7} \mathrm{Mg}_{0.3}$ ) which become a shoulder (less pronounced than for $\mathrm{Li}_{0.7} \mathrm{Mg}_{0.3}$ ) at intermediate $k$ 's [Figs. 6(a) and 2(a)]. This behavior is clearly reflected in the corresponding $S^{N N}(k, \omega)$ spectra. As seen in Figs. 6(b) and 2(b), the $S^{N N}(k, \omega)$ Brillouin peak for $\mathrm{Li}_{0.7} \mathrm{Mg}_{0.3}^{*}$ at $k$ $=0.26 \AA^{-1}$ is less pronounced and shifted towards lower frequencies than that for $\mathrm{Li}_{0.7} \mathrm{Mg}_{0.3}$ while the shoulder at $k$ $=0.74 \AA^{-1}$ for the former system is less visible than for the second. We also calculated the $S_{-}^{11}(k, \omega)$ and $S_{-}^{22}(k, \omega)$ functions at low $k$ 's for $\mathrm{Li}_{0.7} \mathrm{Mg}_{0.3}^{*}$ by using Eq. (2). Unlike for $\mathrm{Li}_{0.7} \mathrm{Mg}_{0.3}$, the frequency of the $S_{-}^{11}(k, \omega)$ and $S_{-}^{22}(k, \omega)$ peaks are still different at the lowest wave vectors explored in this work. Although the frequencies corresponding to light and heavy particles become closer as $k$ decreases they remain different down to wave vectors much lower than in the case of $\mathrm{Li}_{0.7} \mathrm{Mg}_{0.3}$ (Fig. 7). This should be associated with the larger difference of masses of the two species in $\mathrm{Li}_{0.7} \mathrm{Mg}_{0.3}^{*}$. Results in Fig. 7 also corroborate that the peaks and shoulders in the $S^{N N}(k, \omega)$ functions must be mainly associated with the majority Li particles. As in the case of $\mathrm{Li}_{0.7} \mathrm{Mg}_{0.3}$, the sound velocity in $\mathrm{Li}_{0.7} \mathrm{Mg}_{0.3}^{*}$ has been estimated by two routes. On the one hand, according to the scaling law: ${ }^{24}$ $c_{s}^{\mathrm{LiMg} *} \approx c_{s}^{\mathrm{Li}}\left(m_{\mathrm{Li}} / m_{\mathrm{LiMg}} *\right)^{1 / 2}=1.3 \times 10^{3} \mathrm{~m} \mathrm{~s}^{-1}$. On the other hand, through the frequency of the Brillouin peak of $S^{N N}(k, \omega)$ at $k=0.26 \AA^{-1}, \omega^{N N} \approx 3.6 \mathrm{ps}^{-1}$. Thus, $c_{s}^{\mathrm{LiMg}^{*}}$ $\approx 1.4 \times 10^{3} \mathrm{~m} \mathrm{~s}^{-1}$, which is consistent with the value obtained through the scaling law.

\section{LONGITUDINAL CURRENTS}

\section{A. $\operatorname{Li}_{0.7} \mathbf{M g}_{0.3}$ system}

Other properties usually employed to analyze the longitudinal collective modes in liquids are the longitudinal current

intermediate scattering functions (squares and circles) for the $\mathrm{Li}_{0.7} \mathrm{Mg}_{0.3}$ alloy. 

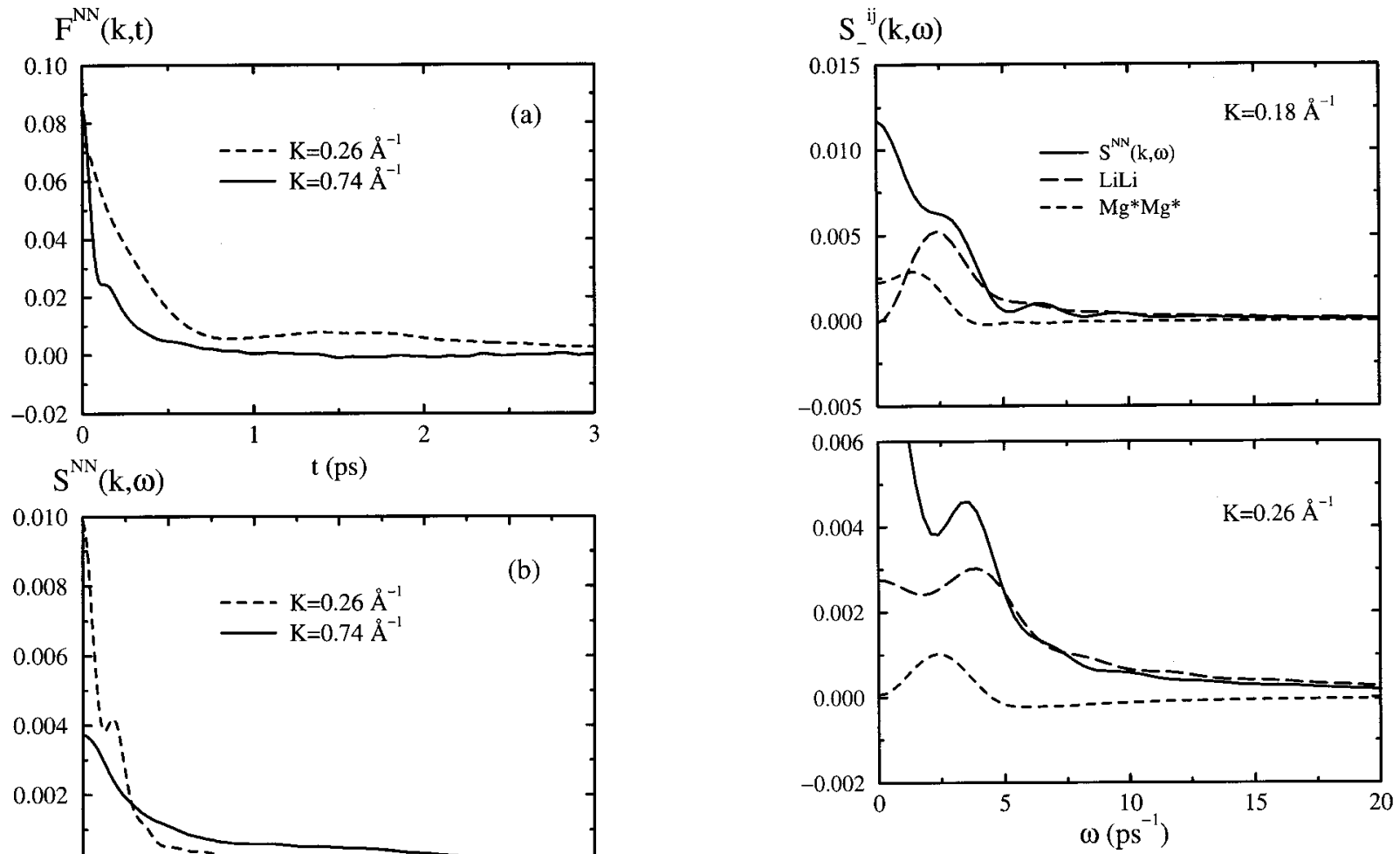

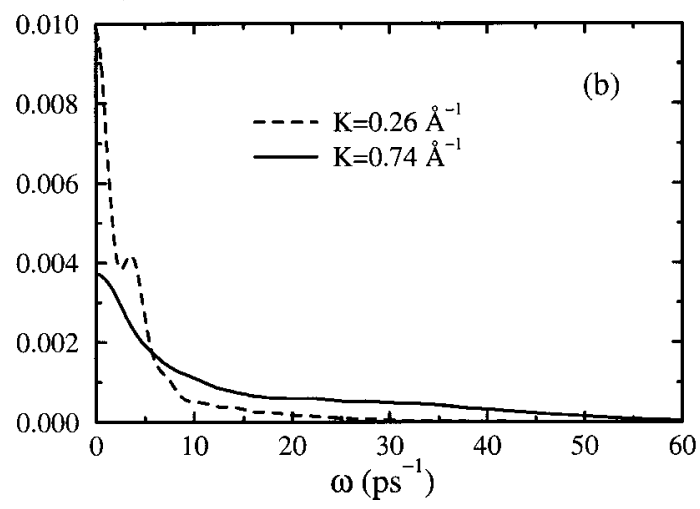

FIG. 6. Number-number intermediate scattering functions (a) and dynamic structure factors (b) for the $\mathrm{Li}_{0.7} \mathrm{Mg}_{0.3}^{*}$ alloy.

correlation functions $C_{L}(k, t)$. The corresponding spectra $C_{L}(k, \omega)$ is related to the dynamic structure factor by ${ }^{15,17}$

$$
C_{L}(k, \omega)=\omega^{2} S(k, \omega) .
$$

According to this relation the Rayleigh component in $S(k, \omega)$ does not show up in $C_{L}(k, \omega)$ whereas the high-frequency part of $S(k, \omega)$ is enhanced. Besides $C_{L}(k, \omega)$ has a visible peak at any $k$. At very low $k$ 's the frequency of this peak is the same as that of the Brillouin peak in $S(k, \omega)$ whereas at higher $k$ 's the frequency of the Brillouin peak, if visible, is somewhat lower. ${ }^{24}$ The partial $C_{L}^{i j}(k, t)$ functions were determined during the simulations while the $C_{L}^{N N}(k, t)$ functions and corresponding spectra $\left[C_{L}^{i j}(k, \omega), C_{L}^{N N}(k, \omega)\right]$ were calculated later. The contributions of both $C_{L}^{22}(k, \omega)$ and $C_{L}^{12}(k, \omega)$ to $C_{L}^{N N}(k, \omega)$ are rather small for all $k$ 's. This shows that $C_{L}^{N N}(k, \omega)$ is largely dominated by correlations of the majority light particles (Fig. 8). $C_{L}^{N N}(k, \omega)$ for $k$ $=0.26 \AA^{-1}$ shows a maximum at a frequency $\left(\omega_{L}^{N N}\right.$ $\approx 10.2 \mathrm{ps}^{-1}$ ) similar but slightly higher than that of the $S^{N N}(k, \omega)$ Brillouin peak. Hence, as with pure liquids, the sound velocity defined through $C_{L}^{N N}(k, \omega)$ is somewhat greater than that through $S^{N N}(k, \omega)$. The longitudinal dispersion relations $\omega_{L}^{N N}(k), \omega_{L}^{11}(k)$, and $\omega_{L}^{22}(k)$ corresponding to $C_{L}^{N N}(k, \omega), C_{L}^{11}(k, \omega)$, and $C_{L}^{22}(k, \omega)$, respectively, are depicted in Fig. 9. $\omega_{L}^{N N}(k)$ shows the same features as $\omega_{L}^{1}(k)$ for pure $\mathrm{Li}$ at similar temperature and density. ${ }^{16}$ So, both $\omega_{L}^{N N}(k)$ and $\omega_{L}^{1}(k)$ have an initial linear increase followed by a maximum at $k \approx 1.3 \AA^{-1}$ and a deep minimum at $k$ $\approx 2.4 \AA^{-1}$ that corresponds to the de Gennes narrowing.
FIG. 7. Corrected partial dynamic structure factors $S_{-}^{11}(k, \omega)$ and $S_{-}^{22}(k, \omega)$ (as defined in the text) and number-number dynamic structure factors $S^{N N}(k, \omega)$ for the $\mathrm{Li}_{0.7} \mathrm{Mg}_{0.3}^{*}$ alloy.

Consistently with the $S_{-}^{i j}(k, \omega)$ findings, the three partial $C_{L}^{i j}(k, \omega)$ functions for $k=0.26 \AA^{-1}$ have a maximum at the same frequency as $C_{L}^{N N}(k, \omega)$ (Fig. 8). This corroborates the hydrodynamic like behavior of light and heavy particles at this $k$. For higher wave vectors, the frequencies of the $C_{L}^{11}(k, \omega)$ maxima increases with $k$ whereas the frequencies of $C_{L}^{22}(k, \omega)$ maxima are significantly lower and remain almost independent of $k$ beyond $k \approx 0.6 \AA^{-1}\left(\omega_{L}^{22} \approx 20 \mathrm{ps}^{-1}\right)$. It should be pointed out that the $\omega_{L}^{11}(k)$ and $\omega_{L}^{N N}(k)$ values are almost identical up to $k \approx 1.5 \AA^{-1}$ and also very similar to the $\omega_{L}^{1}(k)$ values for pure Li. The marked differences between the frequencies for $\mathrm{Li}$ and $\mathrm{Mg}$ atoms suggest that their motions are largely uncorrelated at intermediate wavelengths still far from the kinetic region. The $\omega_{L}^{11}(k)$ function displays a noticeable minimum at $k=2.44 \AA^{-1}$ that should be associated with the de Gennes narrowing. We want to emphasize that for this wave vector $\omega_{L}^{11}(k)$ is quite close to $\omega_{L}^{22}(k)$, while $\omega_{L}^{N N}(k)$ coincides with $\omega_{L}^{22}(k)$ despite the fact that $\mathrm{Li}$ atoms are in the majority in the mixture. These findings show that dynamic collective properties are strongly influenced by the marked spatial correlations at wavelengths corresponding to the maxima of the partial static structure factors.

It is also interesting to investigate the possible existence of propagating concentration modes in simple liquid mixtures. Jacucci and McDonald ${ }^{1}$ did not find evidence of any propagating concentration fluctuation in their former study of the Na-K alloy whereas Jacucci et $a l^{25}$ found side peaks in $S^{C C}(k, \omega)$ for $\mathrm{Li}_{4} \mathrm{~Pb}$ at the same frequencies as in $S^{11}(k, \omega)$. The absence of noticeable concentration modes in Na-K may be attributed to the dominance of the diffusive contributions to the intermediate scattering functions and dynamic struc- 


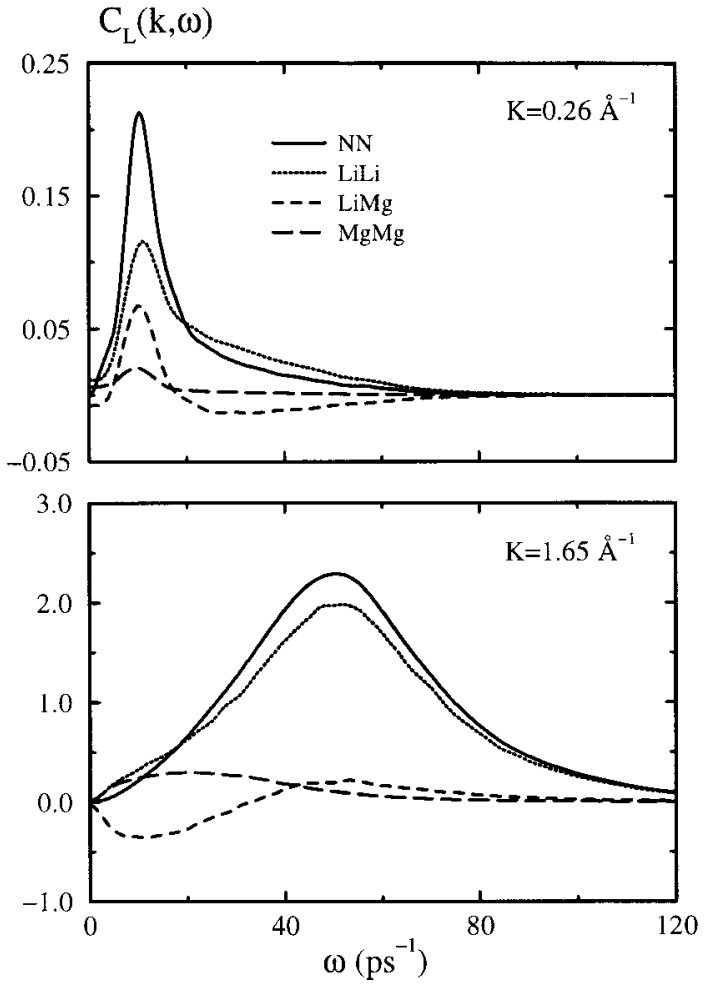

FIG. 8. Partials and number-number longitudinal current spectra for the $\mathrm{Li}_{0.7} \mathrm{Mg}_{0.3}$ alloy.

ture factors. The slowly decaying diffusive contributions are also dominant in $F^{C C}(k, t)$ and $F^{N C}(k, t)$ for $\mathrm{Li}_{0.7} \mathrm{Mg}_{0.3}$. However, since these contributions have little influence on the longitudinal currents, we have analyzed the $C_{L}^{N N}(k, \omega)$, $C_{L}^{C C}(k, \omega)$, and $C_{L}^{N C}(k, \omega)$ functions defined according to Eq. (1). In all cases, $C_{L}^{C C}(k, \omega)$ and $C_{L}^{N C}(k, \omega)$ are much smaller than $C_{L}^{N N}(k, \omega)$ while $C_{L}^{N C}(k, \omega)$ is larger than $C_{L}^{C C}(k, \omega)$. The differences between $C_{L}^{N N}(k, \omega)$ and $C_{L}^{C C}(k, \omega)$ increase as $k$ approaches zero. The resulting $C_{L}^{C C}(k, \omega)$ and $C_{L}^{N C}(k, \omega)$ functions for several representative wave vectors are compared with $C_{L}^{N N}(k, \omega)$ in Fig. 10. For the sake of clarity $C_{L}^{C C}(k, \omega)$ and $C_{L}^{N C}(k, \omega)$ have been

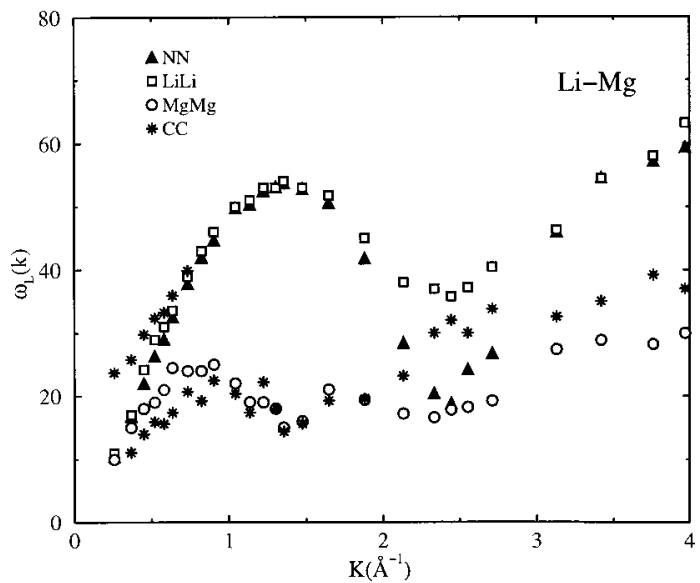

FIG. 9. Dispersion relations of the partials $\left[\omega_{L}^{11}(k), \omega_{L}^{22}(k)\right]$, number-number $\left[\omega_{L}^{N N}(k)\right]$ and concentration-concentration $\left[\omega_{L}^{C C}(k)\right]$ longitudinal modes for the $\mathrm{Li}_{0.7} \mathrm{Mg}_{0.3}$ alloy.

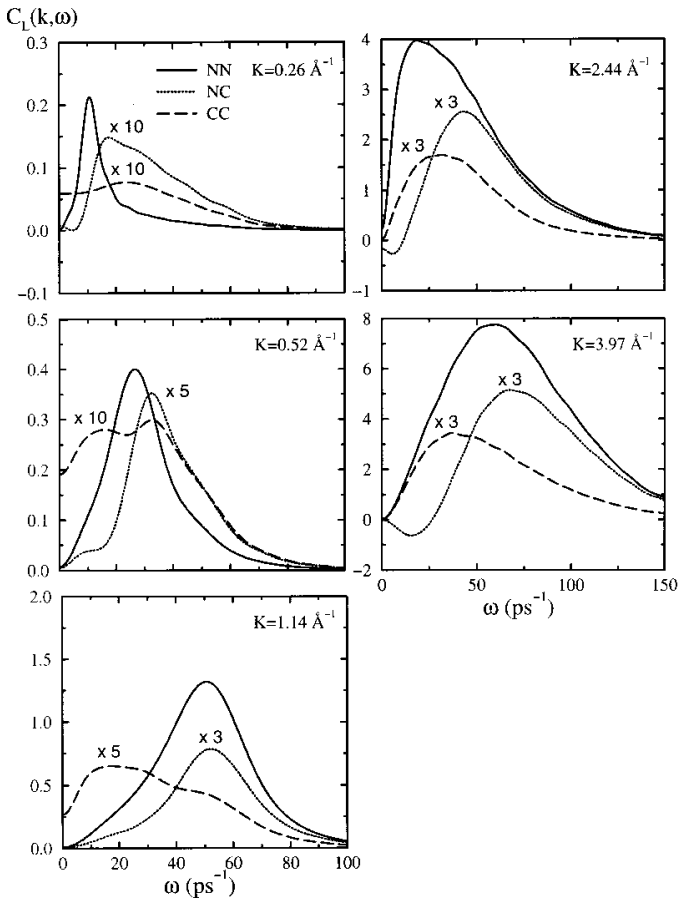

FIG. 10. Number-concentration longitudinal current spectra for the $\mathrm{Li}_{0.7} \mathrm{Mg}_{0.3}$ alloy. For the sake of clarity the $C_{L}^{N C}(k, \omega)$ and $C_{L}^{C C}(k, \omega)$ functions have been multiplied by suitable factors as indicated in the figure.

multiplied by suitable factors. $C_{L}^{C C}(k, \omega)$ shows either one or two peaks that may be associated with propagating concentration modes. At low $k$ ("hydrodynamic region") $C_{L}^{C C}(k, \omega)$ shows only a peak at a frequency higher than that of the $C_{L}^{N N}(k, \omega)$ peak (see the results for $k=0.26 \AA^{-1}$ in Fig. 10). For wave vectors higher than $k \approx 0.4 \AA^{-1}$ two maxima are clearly visible in $C_{L}^{C C}(k, \omega)$, one at a frequency similar though slightly higher than that in $C_{L}^{N N}(k, \omega)$, another at a frequency similar though slightly lower than that in $C_{L}^{22}(k, \omega)$ (see the results for $k=0.52 \AA^{-1}$ in Fig. 10). It may be noticed in Fig. 10 the importance of the $N C$ cross correlations, which could be related to the existence of peaks (or shoulders) at similar frequencies in $C_{L}^{N N}(k, \omega)$ and $C_{L}^{C C}(k, \omega)$ for intermediate $k$ 's.

The dispersion relations corresponding to the $C_{L}^{C C}(k, \omega)$ peaks are illustrated in Fig. 9. It should be pointed out that for $k$ higher than $k \approx 0.8 \AA^{-1}$ the high-frequency maxima become smooth shoulders and only the low-frequency peaks could be plotted in Fig. 9. Beyond $k \approx 2 \AA^{-1}$, the $C C$ modes are very damped and $C_{L}^{C C}(k, \omega)$ shows a broad maximum (Fig. 10) at frequencies intermediate between those of $C_{L}^{11}(k, \omega)$ and $C_{L}^{22}(k, \omega)$. The positions of these $C_{L}^{C C}(k, \omega)$ peaks are not well defined, which leads to rather large uncertainties of the results for this $k$-region displayed in Fig. 9. Moreover, the frequencies of the fast concentration modes tend to a finite value as $k$ goes to zero, which is a characteristic trend of kinetic modes. This corroborates that peaks in $C_{L}^{11}(k, \omega)$ for intermediate $k$ 's, at the same frequencies as those in $C_{L}^{N N}(k, \omega)$ and $C_{L}^{C C}(k, \omega)$, have no acoustic character but should be considered fast kinetic propagating modes. The low-frequency concentration modes at the same frequen- 

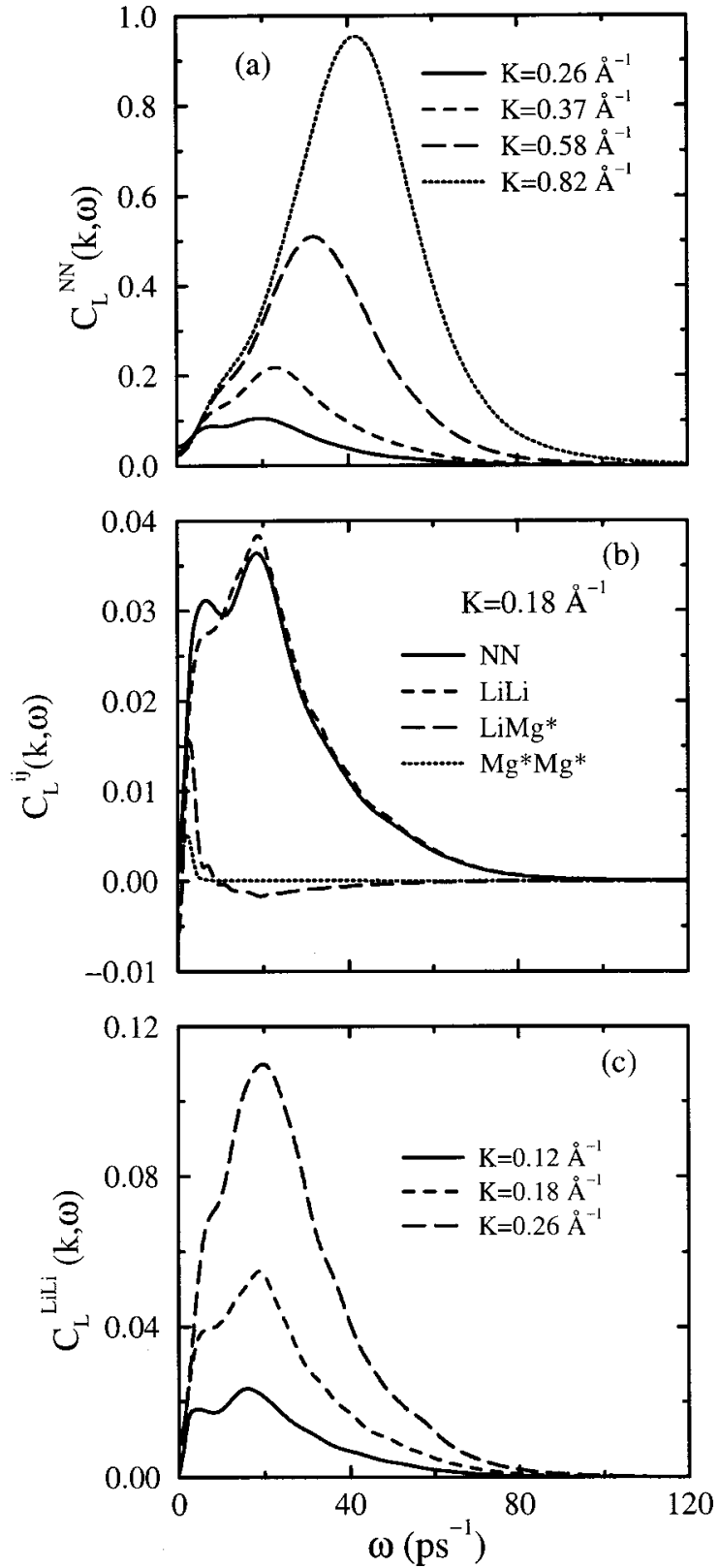

FIG. 11. Number-number and partials longitudinal current spectra for the $\mathrm{Li}_{0.7} \mathrm{Mg}_{0.3}^{*}$ alloy.

cies as in $C_{L}^{22}(k, \omega)$ cannot be observed in the hydrodynamic region, which suggests that these slow propagating modes also have a kinetic character.

\section{B. $\mathrm{Li}_{0.7} \mathrm{Mg}_{0.3}^{*}$ system}

The $C_{L}^{N N}(k, \omega)$ 's for $\mathrm{Li}_{0.7} \mathrm{Mg}_{0.3}^{*}$ have single maxima at frequencies decreasing with $k$ that are very close to those of the corresponding $C_{L}^{11}(k, \omega)$ (Fig. 11). We want to emphasize the existence of a second maximum (or a shoulder) for wave vectors lower than $k \approx 0.3 \AA^{-1}$ that becomes more marked as $k$ decreases [Fig. 11(c)]. Although its frequency is lower than that of the "ordinary" peak and very close to that of $C_{L}^{22}(k, \omega)$, the second peak in $C_{L}^{N N}(k, \omega)$ should be mainly attributed to $C_{L}^{11}(k, \omega)$ [see Fig. 11(b)]. Moreover, the frequency of this peak (or shoulder) agrees with that in $S_{-}^{11}(k, \omega)$ (Fig. 3). The high-frequency peak is hardly visible
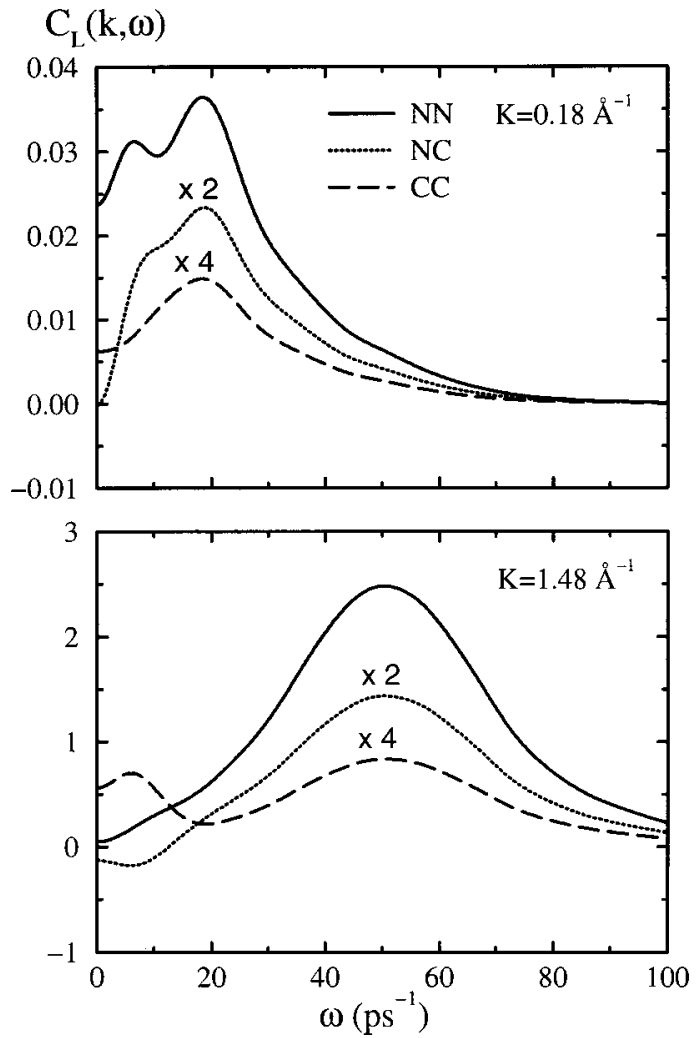

FIG. 12. Number-concentration longitudinal current spectra for the $\mathrm{Li}_{0.7} \mathrm{Mg}_{0.3}^{*}$ alloy. The $C_{L}^{N C}(k, \omega)$ and $C_{L}^{C C}(k, \omega)$ functions have been multiplied by suitable factors as indicated in the figure.

in $S_{-}^{11}(k, \omega)$ but, according to Eq. (3), it is noticeably enhanced in $C_{L}^{11}(k, \omega)$. These findings suggest that during the transition to the hydrodynamic regime $C_{L}^{11}(k, \omega)$ for mixtures of disparate mass particles is the superposition of two functions with peaks at different frequencies.

The resulting $C_{L}^{N N}(k, \omega), C_{L}^{N C}(k, \omega)$, and $C_{L}^{C C}(k, \omega)$ at two representative wave vectors are depicted in Fig. 12. At very low $k$ 's $C_{L}^{C C}(k, \omega)$ shows a high-frequency peak whereas the second peak at lower frequency in $C_{L}^{N N}(k, \omega)$ and $C_{L}^{11}(k, \omega)$ is not visible in this function. However, for $k$ higher than $\approx 0.7 \AA^{-1}$ two maxima may be observed in $C_{L}^{C C}(k, \omega)$. As with $\mathrm{Li}_{0.7} \mathrm{Mg}_{0.3}$ the two frequencies of $C_{L}^{C C}(k, \omega)$ for a given $k$ are very close to those of $C_{L}^{11}(k, \omega)$ and $C_{L}^{22}(k, \omega)$, respectively (Fig. 13). Moreover, the highfrequency concentration modes go to a finite value as $k$ goes to zero whereas the low-frequency concentration modes can only be noticed at $k$ 's quite far from the genuine hydrodynamic region. This confirms that the peaks of the $C_{L}^{C C}(k, \omega)$ functions should be associated with either slow or fast propagating kinetic modes. The $N C$ cross correlations are also important in $\mathrm{Li}_{0.7} \mathrm{Mg}_{0.3}^{*}$ (Fig. 12).

The dispersion relations $\omega_{L}^{11}(k)$ and $\omega_{L}^{22}(k)$ are compared with $\omega_{L}^{N N}(k)$ and $\omega_{L}^{C C}(k)$ in Fig. 13. For the sake of clarity the low frequencies in $C_{L}^{11}(k, \omega)$ for $k$ lower than $\approx 0.3 \AA^{-1}$ are not displayed in this figure. The frequencies corresponding to $\mathrm{Mg}^{*}$ in $\mathrm{Li}_{0.7} \mathrm{Mg}_{0.3}^{*}$ are markedly lower than those for $\mathrm{Mg}$ in $\mathrm{Li}_{0.7} \mathrm{Mg}_{0.3}$, as corresponds to the larger difference between their atomic masses (Figs. 9 and 13). The comparison of the results for identical Li particles in different systems is 


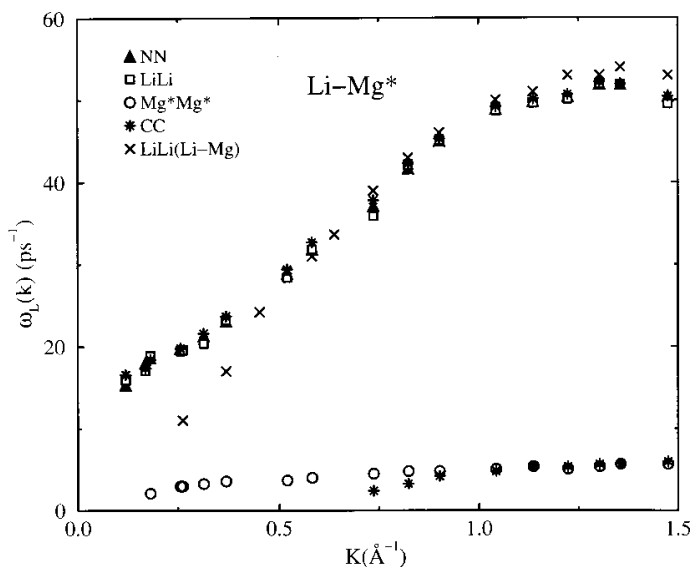

FIG. 13. Dispersion relations of the partials $\left[\omega_{L}^{11}(k), \omega_{L}^{22}(k)\right]$, number-number $\left[\omega_{L}^{N N}(k)\right]$, and concentration-concentration $\left[\omega_{L}^{C C}(k)\right]$ longitudinal modes for the $\mathrm{Li}_{0.7} \mathrm{Mg}_{0.3}^{*}$ alloy. Crosses depict $\omega_{L}^{11}(k)$ for the $\mathrm{Li}_{0.7} \mathrm{Mg}_{0.3}$ alloy.

more interesting. At wave vectors higher than $k \approx 0.5 \AA^{-1}$, $\omega_{L}^{11}(k)$ is almost the same for both $\mathrm{Li}_{0.7} \mathrm{Mg}_{0.3}^{*}$ and $\mathrm{Li}_{0.7} \mathrm{Mg}_{0.3}$ $\left[\omega_{L}^{11}(k)\right.$ for $\mathrm{Li}_{0.7} \mathrm{Mg}_{0.3}$ has been also plotted in Fig. 13] and also very close to $\omega_{L}^{1}(k)$ for pure Li. ${ }^{16}$ This shows that frequencies corresponding to the light particles are independent of the environment down to rather low wave vectors. Moreover, $\omega_{L}^{11}(k)$ and $\omega_{L}^{N N}(k)$ for $\mathrm{Li}_{0.7}-\mathrm{Mg}_{0.3}^{*}$ do not go to zero with $k$, which corroborate the kinetic character attributed to these modes. These findings are consistent with the conclusions of Fernández-Perea et al. ${ }^{9}$ which associated the highfrequency longitudinal modes in $\mathrm{Li}_{4} \mathrm{~Pb}$ (already predicted by kinetic theory ${ }^{8}$ ) with short-lived out-of-phase motions of light atoms.

\section{CONCLUSIONS}

The results obtained in this MD study show that, in general, the $F^{i j}(k, t)$ functions for simple binary liquids have greater initial values and slower time decays than the $F(k, t)$ functions for pure liquids. This should be associated with the dominance of diffusion terms which hide the oscillatory contributions to $F^{i j}(k, t)$ and corresponding peaks in $S^{i j}(k, \omega)$. The main features of the $F^{i j}(k, t)$ and $S^{i j}(k, \omega)$ functions are similar for $\mathrm{Li}_{0.7} \mathrm{Mg}_{0.3}$ and $\mathrm{Li}_{0.7} \mathrm{Mg}_{0.3}^{*}$. This confirms that the existence of clearly visible peaks in $S^{11}(k, \omega)$ for $\mathrm{Li}_{4} \mathrm{~Pb}$ cannot be related only to the large ratio between atomic masses but also to other special features of this system, such as the very different size of the $\mathrm{Li}$ and $\mathrm{Pb}$ particles or the strong deviations from ideal mixing due to the ionic character of this alloy.

The behavior of the $F^{N N}(k, t)$ functions is analogous to that of $F(k, t)$ for pure liquids, showing much lower values than the $F^{i j}(k, t)$ 's and clear oscillations at low $k$ 's, which are reflected in $S^{N N}(k, \omega)$ peaks or shoulders. This is the result of large cancellations between the three $F^{i j}(k, t)$ functions when $F^{N N}(k, t)$ is calculated. Moreover, the de Gennes narrowing typical of one component liquids may be also ob- served in liquid mixtures. The marked static correlations between particles at the wavelength characteristic of the structure of the system have a strong influence on the dynamic collective particles. So, in the case of $\mathrm{Li}_{0.7} \mathrm{Mg}_{0.3}$ the differences between $\omega_{L}^{11}$ and $\omega_{L}^{22}$ are noticeably reduced and $\omega_{L}^{N N}$ becomes almost coincident with $\omega_{L}^{22}$ as $k$ approaches $\approx 2.44$ $\AA^{-1}$ (the wave vector corresponding to the maximum of the static structure factors).

A general trend of liquid binary mixtures at wave vectors prior to the hydrodynamic region is the existence of both fast and slow propagating longitudinal modes, which are associated with light and heavy particles, respectively. The high $\omega^{11}$ and low $\omega^{22}$ frequencies corresponding to these modes cannot generally be observed in the partial $S^{i j}(k, \omega)$ functions (except in special systems such as $\mathrm{Li}_{4} \mathrm{~Pb}$ ) but they can be seen if the diffusive contributions are mostly removed by considering the $C_{L}^{i j}(k, \omega)$ or the $S_{-}^{i j}(k, \omega)$ functions. The frequencies corresponding to the $C_{L}^{N N}(k, \omega)$ peaks are very close to those for the majority light particles. These frequencies are practically independent of the environment down to rather low wave vectors. So, there is a noticeably coincidence between $\omega_{L}^{11}(k)$ for $\mathrm{Li}_{0.7} \mathrm{Mg}_{0.3}^{*}$ and for $\mathrm{Li}_{0.7} \mathrm{Mg}_{0.3}$ as well as with $\omega_{L}^{1}(k)$ for pure Li. Noticeable maxima have also been found in the concentration $C_{L}^{C C}(k, \omega)$ spectra. In most cases these functions show two peaks for a given $k$ at frequencies close to $\omega^{11}$ and $\omega^{22}$, respectively. Our results suggest that both fast and slow propagating modes have a kinetic character, analogous to that of optical modes in ionic solids or molten salts.

In the hydrodynamic limit the high $\left(\omega^{11}, \omega^{N N}\right)$ and low$\left(\omega^{22}\right)$ frequency modes merge into a single acoustic mode. The larger the difference is between the masses of particles in the mixture the lower is the wave number required to reach the hydrodynamic regime. It will be more "difficult" for light particles to oscillate with the same frequency as heavy particles (as they should in the hydrodynamic limit) when there is a large discrepancy between their "natural" frequencies. Although in disparate mass liquid mixtures the high and low frequencies are very different, the transition of $C_{L}^{11}(k, \omega)$ to the hydrodynamic regime is not sharp. So, two peaks may be observed in the $C_{L}^{11}(k, \omega)$ functions for wave vectors approaching the hydrodynamic region. As $k$ decreases the high-frequency peak decreases while the lowfrequency peak increases (the former should disappear at $k$ equal zero). These findings suggest that $C_{L}^{11}(k, \omega)$ is composed of two functions with peaks at different frequencies such that in mixtures of nondisparate mass particles, say $\mathrm{Li}_{0.7} \mathrm{Mg}_{0.3}$, they are so close that they cannot be distinguished.

\section{ACKNOWLEDGMENTS}

The financial support from the DGICYT of the Spanish government (Grant No. PB96-0170-C03-01) and the CIRIT of the "Generalitat de Catalunya" (Grant No. 1999SGR00146) is gratefully acknowledged. 
${ }^{1}$ G. Jacucci and I. R. McDonald, J. Phys. F: Met. Phys. 10, L15 (1980).

${ }^{2}$ J. Bosse, G. Jacucci, M. Ronchetti, and W. Schirmacher, Phys. Rev. Lett. 57, 3277 (1986).

${ }^{3}$ P. H. K. de Jong, P. Verkerk, C. F. de Vroege, L. A. de Graaf, W. S. Howells, and S. M. Bennington, J. Phys.: Condens. Matter 6, L681 (1994).

${ }^{4}$ M. Alvarez, F. J. Bermejo, P. Verkerk, and B. Roessli, Phys. Rev. Lett. 80, 2141 (1998).

${ }^{5}$ W. Montfrooij, P. Westerhuijs, V. O. de Haan, and I. M. de Schepper, Phys. Rev. Lett. 63, 544 (1989); P. Westerhuijs, W. Montfrooij, L. A. de Graaf, and I. M. de Schepper, Phys. Rev. A 45, 3749 (1992); H. E. Smorenburg, R. M. Crevecoeur, and I. M. de Schepper, Phys. Lett. A 211, 118 (1996).

${ }^{6}$ E. Enciso, N. G. Almarza, P. Dominguez, M. A. González, and F. J. Bermejo, Phys. Rev. Lett. 74, 4233 (1995).

${ }^{7}$ T. Bryk, I. Mryglod, and G. Kahl, Phys. Rev. E 56, 2903 (1997).

${ }^{8}$ A. Campa and E. G. D. Cohen, Phys. Rev. A 41, 5451 (1990), and references therein.

${ }^{9}$ R. Fernández-Perea, M. Alvarez, F. J. Bermejo, P. Verkerk, B. Roessli, and E. Enciso, Phys. Rev. E 58, 4568 (1998).

${ }^{10}$ E. Enciso, N. G. Almarza, M. A. González, F. J. Bermejo, R. Fernández-Perea, and F. Bresme, Phys. Rev. Lett. 81, 4432 (1998).

${ }^{11}$ A. P. Copestake, R. Evans, H. Ruppersberg, and W. Schirmacher, J. Phys. F: Met. Phys. 13, 1993 (1983).
${ }^{12}$ H. Ruppersberg and W. Knoll, Z. Naturforsch. A 32, 1374 (1977).

${ }^{13}$ M. Canales, D. J. González, L. E. González, and J. A. Padró, Phys. Rev. E 58, 4747 (1998).

${ }^{14}$ L. E. González, D. J. González, M. Silbert, and J. A. Alonso, J. Phys.: Condens. Matter 5, 4283 (1993).

${ }^{15}$ M. Canales, J. A. Padró, L. E. González, and A. Giró, J. Phys.: Condens. Matter 5, 3095 (1993).

${ }^{16}$ M. Canales, L. E. González, and J. A. Padró, Phys. Rev. E 50, 3656 (1994).

${ }^{17}$ N. Anento, J. Casas, M. Canales, D. J. González, L. E. González, and J. A. Padró, J. Non-Cryst. Solids 250-252, 348 (1999).

${ }^{18}$ J. P. Hansen and I. R. McDonald, Theory of Simple Liquids (Academic, London, 1986).

${ }^{19}$ U. Balucani and M. Zoppi, Dynamics of the Liquid State (Oxford University Press, Oxford, 1994).

${ }^{20}$ N. H. March and M. P. Tosi, Atomic Dynamics in Liquids (McMillan, London, 1976).

${ }^{21}$ R. Vogelsang, C. Hoheisel, G. V. Paolini, and G. Ciccotti, Phys. Rev. A 36, 3964 (1987).

${ }^{22}$ R. D. Mountain and J. M. Deutch, J. Chem. Phys. 50, 1103 (1969).

${ }^{23}$ Ya. Chushak and A. Baumketner, Eur. Phys. J. B 7, 129 (1999).

${ }^{24}$ J. P. Boon and S. Yip, Molecular Hydrodynamics (McGraw-Hill, New York, 1980).

${ }^{25}$ G. Jacucci, M. Ronchetti, and W. Schirmacher, J. Phys. (Paris), Colloq. 12, C8-385 (1985). 